\section{Dr. J. H. Wildhaber ist der erste Preisträger des "Swiss Medical Weekly Young Investigator's Award»}

25000 Franken für die Forschung: Nicht nur eine grossartige Idee, sondern auch ein substantieller finanzieller Beitrag! Die Redaktion der Schweizerischen Medizinischen Wochenschrift hatte den guten Einfall, die Geschäftsleitung des Verlages EMH unterstützte ihn und setzte ihn um. Dies könnte nun ein wirklich gutes Omen für die jungen Forscherinnen und Forscher sein: im eigenen Land für die erreichten wissenschaftlichen Leistungen anerkannt zu werden ...

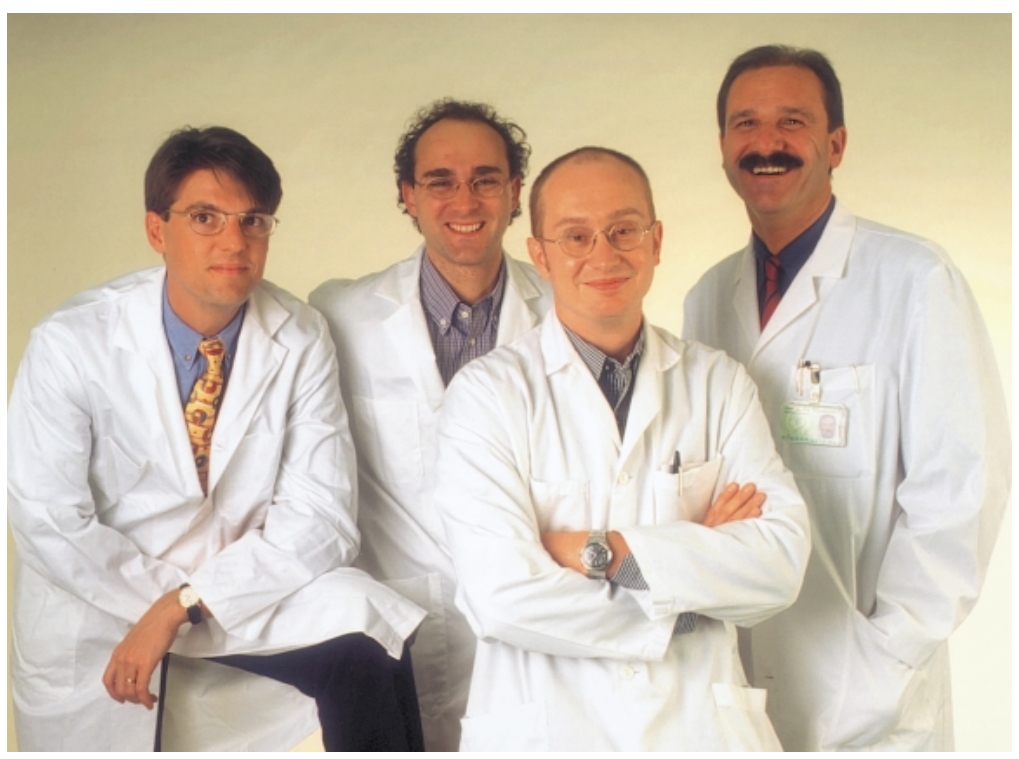

Pneumologisches Forschungsteam der Universitätsklinik Zürich: G. L. Hall, A. Möller, J. H. Wildhaber, F. H. Sennhauser (v.l.n.r.)

Originalpublikation: Schweiz Med Wochenschr 2000;130:529-34. Internet: http://www.smw.ch/archive/2000/130-15-374-00.html Volltext als pdf-File unter http://www.smw.ch/pdf/2000_15/2000-15-374.pdf
Der erste Preisträger des «Swiss Medical Weekly Young Investigator's Award" ist Dr. Johannes H. Wildhaber vom Kinderspital Zürich. 15 Manuskripte sind für den Preis eingereicht worden, und wir sind allen Forscherinnen und Forschern, die sich beteiligt haben, zu Dank verpflichtet. Wir haben Arbeiten mit ausgezeichnetem Studiendesign entdeckt, sehr originelle Ideen und viele relevante Daten. Nur ganz wenige Arbeiten mussten abgelehnt werden, die meisten waren von beeindruckender wissenschaftlicher Qualität, ein Spiegelbild der aktuellen Forschungstätigkeit in der Schweiz.

Dr. Wildhaber erhält den SMW-Preis für die Arbeit:

Levels of exhaled nitric oxide in recurrently wheezy infants are decreased following inhaled steroid therapy (Wildhaber JH, Möller A, Hall GL, Sennhauser F, Stick SM)

Die Autoren konnten zeigen, dass ausgeatmetes Stickstoffmonoxid mit der für das Asthma typischen Entzündung der Atemwege korreliert und dass die Konzentration des ausgeatmeten Stickstoffmonoxids nach Therapie mit inhalierbaren Steroiden sinkt. Ähnliche Daten sind kürzlich für Erwachsene publiziert worden, die Daten von Wildhaber et al. sind aber neu für kleine Kinder. Der wirklich neuartige Aspekt dieser Arbeit ist die Erarbeitung von neuen Techniken, die es erlauben, solche Prozesse bei sehr kleinen Kindern zu dokumentieren. Die Studie wurde im Kinderspital Zürich durchgeführt, in enger Zusammenarbeit mit dem «Princess Margaret Hospital for Children» in Perth, Australien, wo der Preisträger drei Jahre verbracht und sowohl Grundlagenforschung als auch klinische Forschung betrieben hat.

Die Preisverleihung findet am 29. Juni 2000 im Rahmen des ärztlichen Fortbildungsprogramms der Universitäts-Kinderklinik Zürich (im Hörsaal des Kinderspitals) statt.

16.30 Uhr: Fallvorstellungen aus der Pädiatrischen Klinik (Dr. U. Lips)

17.20 Uhr: Vorstellung der preisgekrönten Arbeit (Dr. J. Wildhaber)

17.40 Uhr: Laudatio und Preisübergabe

(Prof. A. P. Perruchoud)

Redaktion und Verlag der SMW freuen sich, bekanntgeben zu können, dass der "Swiss Medical Weekly Young Investigator's Award" jedes Jahr verliehen werden wird, solange exzellente Forschungsarbeiten eingereicht werden. Wir zweifeln nicht daran, dass junge Kolleginnen und Kollegen in der Schweiz gute wissenschaftliche Forschung betreiben, und wir freuen uns bereits auf die nächste Preisrunde. Forscherinnen und Forscher, macht Euch an die Arbeit!

Für die Redaktion der SMW Prof. Dr. med. André P. Perruchoud 